\title{
ADSORÇÃO DE SELÊNIO EM LATOSSOLOS ${ }^{(1)}$
}

\author{
Ernesto Rinaldi Mouta ${ }^{(2)}$, Wanderley José de Melo ${ }^{(3)}$, Marcio \\ Roberto Soares $^{(4)}$, Luis Reynaldo Ferracciú Alleoni ${ }^{(5)}$, \& José \\ Carlos Casagrande ${ }^{(4)}$
}

\begin{abstract}
RESUMO
A retenção de Se pelos colóides do solo constitui importante processo para a manutenção da sanidade ambiental. A informação sobre a adsorção de Se em solos altamente intemperizados é restrita e existem poucos padrões quantitativos disponíveis para a definição de estratégias de remediação de áreas contaminadas. Quantidades crescentes de $\mathrm{Se}\left(5,10,25,50,100\right.$ e $\left.250 \mathrm{mg} \mathrm{L}^{-1}\right)$, na forma de $\mathrm{Na}_{2} \mathrm{SeO}_{3}$, foram adicionadas a amostras de dez Latossolos brasileiros [três Latossolos Vermelho-Amarelos (LVA-1, LVA-2 e LVA-3), dois Latossolos Vermelhos (LV-1 e LV-2), um Latossolo Vermelho eutroférrico (LVef), um Latossolo Vermelho acriférrico (LVwf), dois Latossolos Amarelos (LA-1 e LA-2) e um Latossolo Amarelo acriférrico (LAwf)]. Isotermas de adsorção foram construídas e foi verificado o ajuste dos resultados experimentais aos modelos de Langmuir e de Freundlich. A equação de Langmuir ajustou melhor os resultados de adsorção de Se do que a isoterma de Freundlich. Todas as isotermas apresentaram o formato tipo-L (exponencial), com exceção daquelas obtidas para o LVA-1 e para o LVA-2, que apresentaram comportamento tipo-C (linear). Valores de adsorção máxima (Ads $s_{\text {máx }}$ ), estimada pelo modelo de Langmuir, variaram de 135 (LVA-3) a $2.245 \mathrm{mg} \mathrm{kg}^{-1}$ (LA-1), enquanto os coeficientes de afinidade $\left(\mathrm{K}_{\mathrm{L}}\right)$ estiveram entre 0,002 (LVA-2) e 0,326 (LVA-3). A constante de afinidade estimada pelo modelo de Freundlich $\left(\mathrm{K}_{\mathrm{f}}\right)$ variou de 13,7 (LVA-2) a 180,1 (LAwf). A adsorção máxima de Se foi mais elevada no LVef e nos Latossolos ácricos (LAwf e LVwf), enquanto os maiores valores de $K_{f}$ foram encontrados no LV-2, LVef, LVA-3 e LVwf. Não houve
\end{abstract}

\footnotetext{
${ }^{(1)}$ Parte da Tese de Mestrado do primeiro autor, apresentada ao Programa de Pós- Graduação em Ciência do Solo, Faculdade de Ciências Agrárias e Veterinárias, Universidade Estadual Paulista - FCAV/UNESP. Recebido para publicação em junho de 2006 e aprovado em março de 2008.

(2) Mestre em Ciência do Solo pela Faculdade de Ciências Agrárias e Veterinárias, Universidade Estadual Paulista - FCAVUNESP. CEP 14884-900 Jaboticabal (SP). E-mail: ermouta@yahoo.com.br

(3) Professor do Departamento de Tecnologia da FCAV-UNESP. Pesquisador do CNPq. E-mail: wjmelo@fcav.unesp.br

(4) Professor do Departamento de Recursos Naturais e Proteção Ambiental, Universidade Federal de São Carlos - UFSCar. Rodovia Anhanguera, km 174, Caixa Postal 153, CEP 13600-970 Araras (SP). E-mail: mrsoares@cca.ufscar.br; bighouse@power.ufscar.br;

(5) Professor do Departamento de Ciência do Solo, Escola Superior de Agricultura "Luiz de Queiroz" - ESALQ. Caixa Postal 09, Av. Pádua Dias 11, CEP 13418-900 Piracicaba (SP). E-mail: alleoni@esalq.usp.br
} 
correlação entre os atributos dos solos e as constantes de Langmuir. Valores de $\mathbf{K}_{\mathbf{f}}$ correlacionaram-se com os teores de argila $\left(r=0,42^{*}\right)$ e com a capacidade de troca de ânions $(r=0,64 *)$.

Termos de indexação: isotermas, Langmuir, Freundlich, solos tropicais.

\section{SUMMARY: SELENIUM ADSORPTION IN OXISOLS}

Selenium (Se) retention by soil colloids is an important process to maintain the environmental quality. Little information is available on Se adsorption in highly weathered tropical soils, and there are few numerical parameters that can be used as references to aid on adoption management strategies in contaminated areas. Increasing amounts of Se (5, $10,25,50,100$ and $250 \mathrm{mg} \mathrm{L}^{-1}$ ), added as $\mathrm{Na}_{2} \mathrm{SeO}_{3}$, were equilibrated with samples of ten Brazilian Oxisols [three Typic Hapludoxes (TH-1, TH-2 and TH-3), two Rhodic Eutrudoxes (RE-1 and RE-2), two Xanthic Hapludoxes (XH-1 and $\mathrm{XH}-2)$, one Rhodic Hapludox (RH), one Rhodic Acrudox (RA) and one Anionic Acrudox (AA)]. Adsorption isotherms were obtained and the experimental results fitted to Langmuir and Freundlich models. The Langmuir equation fitted better to the experimental data than the Freundlich model. All isotherms had an L-type (exponential) shape, except for TH-1 and TH-2, which had a $C$ type (linear) shape. Selenium Ads $s_{\max }$ varied between $135 \mathrm{mg} \mathrm{kg}^{-1}$ (TH-3) and $2.245 \mathrm{mg} \mathrm{kg}^{-1}$ $(\mathrm{XH}-1)$ and the affinity constant $\left(\mathrm{K}_{\mathrm{L}}\right)$ varied from $0.002(\mathrm{TH}-2)$ to $0.326 \mathrm{~L} \mathrm{~kg}^{-1}$ (TH-3). Affinity constant $\left(K_{f}\right)$ values between $13.7(T H-2)$ and $180.1 \mathrm{~L} \mathrm{~kg}^{-1}(A A)$ were obtained from the Freundlich model. A high Se retention potential was observed on $R H$ and on acric Oxisols (AA and RA), while qualitative parameters indicated $R E-2, R H, T H-3$ and $R A$ as Oxisols with high Se affinity. No correlations were observed between soil attributes and Langmuir parameters. The Freundlich coefficient $\left(K_{f}\right)$ correlated with clay content $\left(r=0.42^{*}\right)$ and with anion exchange capacity $(A E C)\left(r=0.64^{*}\right)$.

Index terms: isotherms, Langmuir, Freundlich, tropical soils.

\section{INTRODUÇÃO}

O Se é um elemento não-metálico encontrado naturalmente na crosta terrestre em concentrações que variam de 0,1 a até $1.000 \mathrm{mg} \mathrm{kg}^{-1}$, em áreas de solos naturalmente ricos em Se(Fishbein, 1991). Esses teores correspondem à fração litogênica e fazem parte dos ciclos biogeoquímicos naturais ao serem liberados das estruturas cristalinas das rochas por processos de intemperização. Embora os constituintes do solo condicionem os teores de Se neste meio, atividades antropogênicas contribuem para o aumento das taxas de transferência desse elemento entre os diversos compartimentos dos ecossistemas. O Se tem sido utilizado extensivamente pela sociedade moderna industrializada como elemento principal na confecção de equipamentos eletrônicos e fotográficos, em artigos de impressão, na xerografia, na formulação de reagentes químicos, plásticos, lubrificantes e nas indústrias cosméticas e farmacêuticas (Lisk, 1972; Girling, 1984; Dhillon \& Dhillon, 2003). O Se também é componente de insumos agropecuários, como defensivos agrícolas e fertilizantes, especialmente os fosfatados (Lisk, 1972; Girling, 1984), e está presente em suplementos alimentares, por ser essencial à nutrição animal (Lisk, 1972; Bar-Yosef \& Meek, 1987; Neal, 1995).

A disposição inadvertida de resíduos urbanos e industriais (Dhillon \& Dhillon 2003) e a utilização inadequada de defensivos agrícolas e fertilizantes (Lisk, 1972) têm gerado aumento na quantidade de Se liberado nos solos, o que facilita seu ingresso na cadeia alimentar. Sua mobilidade e biodisponibilidade dependem do aumento da sua concentração na solução do solo, de onde o elemento pode ser absorvido e translocado pelas plantas ou pode ser lixiviado até os aqüíferos subterrâneos. Sua persistência no solo e a atenuação de sua mobilidade envolvem fenômenos de sorção, dessorção, precipitação, complexação, oxirredução e dissolução (Sparks, 1999). Tais reações podem ocorrer simultaneamente, mas os mecanismos de adsorção são determinantes no controle da disponibilidade e da solubilidade de contaminantes (Ford et al., 2001).

No solo, encontra-se especialmente sob as formas aniônicas de selenito $\left(\mathrm{SeO}_{3}^{2-}\right)$ e de selenato $\left(\mathrm{SeO}_{4}{ }^{2-}\right)$. No entanto, a forma elementar $\left(\mathrm{Se}^{0}\right)$ e a forma orgânica também ocorrem. À medida que seu estado de oxidação é aumentado, o Se torna-se mais móvel e menos retido no solo (Neal, 1995). Em regiões de alta umidade e, 
ou, de solos ácidos, selenito é a espécie química predominante (Neal, 1995; Nakamaru et al., 2005). Sob condições de neutralidade ou alcalinidade, selenito tende à oxidação, passando para a forma de selenato (Girling, 1984; Geering et al., 1968). A maioria dos estudos sobre adsorção de Se foi realizada em sistemas puros, como os oxihidróxidos de ferro cristalinos [goethita (Balistrieri \& Chao, 1987)] e amorfos (Balistrieri \& Chao, 1990), os de manganês (Balistrieri \& Chao, 1990; Saeki et al., 1995) e os de alumínio (Peak, 2006), assim como em filossilicatos, o que inclui a caulinita e a montmorilonita (Bar-Yosef \& Meek, 1987). Os oxihidróxidos de ferro são reconhecidamente a fração mais ativa na adsorção de espécies aniônicas de Se (Balistrieri \& Chao, 1987). Existe pouca informação sobre o comportamento de Se em solos (Nakamaru et al., 2005), sobretudo naqueles da região tropical úmida.

As isotermas de adsorção descrevem matematicamente a relação entre a concentração adsorvida e aquela em solução (Sposito, 1989). O formato e o ajuste matemático de isotermas de adsorção fornecem informações importantes sobre a capacidade de adsorção e sobre a força pela qual o adsorvato está retido no solo. Em solos tropicais, as isotermas de Langmuir e de Freundlich têm sido utilizadas para ajustar resultados da adsorção de ânions como o fosfato (Casagrande et al., 2003), o sulfato (Casagrande et al., 2003; Alves \& Lavorenti, 2004) e o borato (Alleoni et al., 1998; Soares et al., 2005). Todavia, os parâmetros de avaliação para o Se ainda são escassos para solos brasileiros.

O objetivo deste trabalho foi avaliar a correlação entre os atributos de dez Latossolos do Estado de São Paulo e os parâmetros quantitativos da reação de adsorção de Se obtidos a partir do ajuste dos modelos de Langmuir e de Freundlich aos resultados experimentais.

\section{MATERIAL E MÉTODOS}

\section{Amostras de solos}

Com vistas em obter ampla variação dos atributos químicos, físicos e mineralógicos da classe de solos mais importante no Brasil, foram utilizadas amostras superficiais $(0-0,2 \mathrm{~m})$ de dez Latossolos representativos do Estado de São Paulo [três Latossolos Vermelho-Amarelos (LVA-1, LVA-2 e LVA-3), dois Latossolos Vermelhos (LV-1 e LV-2), um Latossolo Vermelho eutroférrico (LVef), um Latossolo Vermelho acriférrico (LVwf), dois Latossolos Amarelos (LA-1 e LA-2) e um Latossolo Amarelo acriférrico (LAwf)], coletadas em áreas isentas de ações antrópicas e caracterizadas por Soares (2004) (Quadro 1).

\section{Quadro 1. Localização geográfica e resultados das análises dos atributos químicos e físicos dos solos}

\begin{tabular}{|c|c|c|c|c|c|c|c|c|c|c|c|c|c|c|c|}
\hline Solo & $\begin{array}{c}\text { Coord. } \\
\text { geográfica }\end{array}$ & $\begin{array}{c}\mathrm{pH} \\
\mathrm{H}_{2} \mathrm{O}\end{array}$ & $\Delta \mathbf{p H}$ & $\mathrm{CO}$ & $\mathrm{CTC}_{\mathrm{t}}$ & $\mathrm{CTC}_{\mathrm{e}}$ & CTA & Fedcв & Feox & $\mathrm{Al}_{\mathrm{x}}$ & Mnox & $\mathrm{MnO}$ & Areia & Silte & Argila \\
\hline & & & & $\mathrm{g} \mathrm{kg}^{-1}$ & \multicolumn{3}{|c|}{$\longrightarrow \mathrm{mmol}_{\mathrm{c}} \mathrm{kg}^{-1}$} & \multicolumn{7}{|c|}{$-\mathrm{g} \mathrm{kg}^{-1}$} & 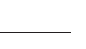 \\
\hline LVA-1 & $\begin{array}{c}22^{\circ} 19^{\prime} \mathrm{S} \\
47^{\circ} 10^{\prime} \mathrm{W}\end{array}$ & 4,5 & $-1,15$ & 12,4 & 16,9 & 12,3 & 1,9 & 19,30 & 1,72 & 0,81 & 0,01 & 0,02 & 779 & 40 & 181 \\
\hline LVA-2 & $\begin{array}{c}22^{\circ} 15^{\prime} \mathrm{S} \\
47^{\circ} 49^{\prime} \mathrm{W}\end{array}$ & 4,2 & $-1,02$ & 29,9 & 29,9 & 15,5 & 2,1 & 13,59 & 0,85 & 0,49 & 0,01 & 0,04 & 759 & 20 & 221 \\
\hline LVA-3 & $\begin{array}{l}22^{\circ} 19^{\prime} \mathrm{S} \\
47^{\circ} 10^{\prime} \mathrm{W}\end{array}$ & 4,3 & $-1,00$ & 16,4 & 20,6 & 11,3 & 2,3 & 21,32 & 1,58 & 1,14 & 0,02 & 0,06 & 738 & 60 & 202 \\
\hline LV-1 & $\begin{array}{l}22^{\circ} 01^{\prime} \mathrm{S} \\
47^{\circ} 53^{\prime} \mathrm{W}\end{array}$ & 6,4 & $-1,35$ & 19,4 & 36,9 & 28,4 & 2,8 & 53,13 & 3,07 & 1,59 & 0,04 & 0,72 & 718 & 81 & 201 \\
\hline LV-2 & $\begin{array}{l}22^{\circ} 43^{\prime} \mathrm{S} \\
47^{\circ} 38^{\prime} \mathrm{W}\end{array}$ & 4,5 & $-1,18$ & 38,1 & 59,3 & 30,6 & 3,7 & 86,58 & 6,36 & 3,97 & 0,16 & 1,63 & 368 & 102 & 530 \\
\hline Lvef & $\begin{array}{l}21^{\circ} 05^{\prime} \mathrm{S} \\
47^{\circ} 08^{\prime} \mathrm{W}\end{array}$ & 7,2 & $-0,56$ & 95,6 & 62,2 & 60,4 & 3,2 & 185,16 & 14,87 & 8,61 & 1,14 & 13,08 & 109 & 207 & 684 \\
\hline LVwf & $\begin{array}{l}21^{\circ} 10^{\prime} \mathrm{S} \\
47^{\circ} 48^{\prime} \mathrm{W}\end{array}$ & 4,7 & $-0,93$ & 67,4 & 58,4 & 23,7 & 3,8 & 208,17 & 19,25 & 9,81 & 0,51 & 5,64 & 141 & 143 & 716 \\
\hline LA-1 & $\begin{array}{l}22^{\circ} 15^{\prime} \mathrm{S} \\
47^{\circ} 49^{\prime} \mathrm{W}\end{array}$ & 4,8 & $-0,86$ & 38,8 & 44,2 & 23,4 & 3,0 & 19,74 & 1,34 & 0,72 & 0,06 & 0,97 & 738 & 40 & 222 \\
\hline LA-2 & $\begin{array}{c}21^{\circ} 57^{\prime} \mathrm{S} \\
47^{\circ} 59^{\prime} \mathrm{W}\end{array}$ & 4,4 & $-0,90$ & 20,1 & 23,4 & 10,5 & 2,2 & 42,16 & 3,45 & 2,55 & 0,03 & 0,28 & 597 & 60 & 342 \\
\hline Lawf & $\begin{array}{l}20^{\circ} 10^{\prime} \mathrm{S} \\
48^{\circ} 02^{\prime} \mathrm{W}\end{array}$ & 4,7 & $-0,97$ & 39,9 & 47,8 & 16,9 & 3,9 & 113,32 & 7,68 & 5,45 & 0,12 & 1,19 & 407 & 123 & 470 \\
\hline
\end{tabular}

$\overline{\mathrm{pH}}$ : medido em água; $\Delta \mathrm{pH}=\mathrm{pH}_{\mathrm{KCl}}-\mathrm{pH}_{\text {água }} ; \mathrm{CO}$ : método titulométrico; capacidade de troca catiônica efetiva $(\mathrm{CTC}=\mathrm{CB}+\mathrm{Al}) \mathrm{e}$ a total $\left(\mathrm{CTC}_{\mathrm{t}}=\mathrm{CB}+(\mathrm{H}+\mathrm{Al})\right)$ estimadas pelo método indireto; capacidade de troca aniônica (CTA) determinada pelo método direto da troca compulsiva; $\mathrm{Fe}_{\mathrm{DCB}}=$ teor de ferro extraído em solução ditionito-citrato-bicarbonato de sódio; $\mathrm{Fe}_{\mathrm{ox}}$, $\mathrm{Al}_{\mathrm{ox}}$ e $\mathrm{Mn}_{\mathrm{ox}}=$ teores de ferro, alumínio e manganês extraídos em solução de oxalato de amônio (reagente Tamm); MnO = fração facilmente redutível de óxidos de manganês extraída em solução de hidrocloreto de hidroxilamina; areia, silte e argila quantificados pelo método do densímetro. Fonte: Soares (2004). 


\section{Caracterização dos solos}

A caracterização química e física dos solos foi feita em amostras de terra fina seca ao ar (TFSA), passadas em peneira de malha de $2 \mathrm{~mm}$, conforme procedimentos descritos em Camargo et al. (1986). O $\mathrm{pH}$ foi potenciometricamente determinado em suspensões de $\mathrm{H}_{2} \mathrm{O}$ e de $\mathrm{KCl} 1 \mathrm{~mol} \mathrm{~L}^{-1}$ com relação solo:solução 1:2,5. A acidez total $(\mathrm{H}+\mathrm{Al})$ foi determinada em solução de acetato de cálcio $1 \mathrm{~mol} \mathrm{~L}^{-1}$ com posterior titulação com hidróxido de amônio 0,025 mol L-1. Os teores de carbono orgânico (CO) foram quantificados por oxidação da matéria orgânica com solução de dicromato de potássio, na presença de ácido sulfúrico e titulação do excesso de dicromato com íons $\mathrm{Fe}^{2+}$ obtidos a partir de uma solução padronizada de sulfato ferroso amoniacal. Cátions básicos (CB) foram extraídos por resina trocadora de íons, sendo Ca e $\mathrm{Mg}$ determinados por espectrofotometria de absorção atômica e $\mathrm{Na}$ e $\mathrm{K}$ por fotometria de emissão de chama. O alumínio trocável foi extraído por solução de cloreto de potássio $1 \mathrm{~mol} \mathrm{~L}^{-1}$ e determinado por titulação com hidróxido de amônio $0,025 \mathrm{~mol} \mathrm{~L}^{-1}$. A capacidade de troca catiônica efetiva $\left(\mathrm{CTC}_{\mathrm{e}}=\mathrm{CB}+\mathrm{Al}\right)$ e a total $\left[\mathrm{CTC}_{t}\right.$ $=\mathrm{CB}+(\mathrm{H}+\mathrm{Al})]$ foram estimadas indiretamente pelo método da soma. A capacidade de troca aniônica (CTA) foi determinada diretamente pelo $\mathrm{pH}$ do solo, a partir do método da troca compulsiva, usando solução de cloreto de bário não tamponada (Gillman et al., 1979). Teores de óxidos de $\mathrm{Fe}, \mathrm{Al}$ e $\mathrm{Mn}\left(\mathrm{Fe}_{\mathrm{ox}}, \mathrm{Al}_{\mathrm{ox}}\right.$ e $\left.\mathrm{Mn}_{\mathrm{ox}}\right)$ mal cristalizados ("amorfos") foram extraídos por solução ácida de oxalato de amônio (Reagente de Tamm). Formas cristalinas ( $\mathrm{Fe}_{\mathrm{DCB}}$ ) de óxidos de $\mathrm{Fe}$ foram extraídas por soluções de ditionito-citratobicarbonato de sódio (DCB-Na). A fração facilmente redutível dos óxidos de $\mathrm{Mn}$ foi determinada pelo método da dissolução seletiva, usando hidrocloreto de hidroxilamina como extrator (Gambrell, 1996). A análise granulométrica foi feita pelo método do densímetro que se baseia na velocidade de decantação das diferentes partículas do solo.

\section{Estudos de adsorção}

Experimentos de adsorção em batelada (tipo "batch de laboratório") foram realizados para a construção de isotermas de adsorção, elaboradas para amostras de terra ao $\mathrm{pH}$ natural. A 2,0 g de terra fina seca ao ar foram adicionados $20 \mathrm{~mL}$ de solução eletrolítica suporte 0,01 mol L-1 de $\mathrm{NaNO}_{3}$ (relação 1:10), que continham 5; 10; 25; 50; 100 e $250 \mathrm{mg} \mathrm{L}^{-1}$ de Se, adicionados na forma de selenito de sódio anidro $\left(\mathrm{Na}_{2} \mathrm{O}_{3} \mathrm{Se}\right)$, em triplicata. O conjunto foi acondicionado em frasco de polietileno e colocado sob agitação $\left( \pm 120\right.$ ciclos min $^{-1}$ ) por $24 \mathrm{~h}$ em agitador pendular. Após a agitação e subseqüente repouso, uma alíquota de $8 \mathrm{~mL}$ foi tomada e levada a outro recipiente para ultracentrifugação a $14.000 \mathrm{rpm}$ por $10 \mathrm{~min}$. A suspensão foi filtrada, e a concentração de Se remanescente no sobrenadante foi determinada por espectrometria de absorção atômica.
A quantidade de Se adsorvido foi computada pela relação $A d s=\left[\left(C_{0}-C_{e q}\right) V\right] / M$, em que $A d s$ é a quantidade de Se adsorvido após o equilíbrio $\left(\mathrm{mg} \mathrm{kg}^{-1}\right)$; $C_{0}$ e $C_{e q}$ são as concentrações de Se inicialmente adicionada e a de equilíbrio $\left(\mathrm{mg} \mathrm{L}^{-1}\right)$, respectivamente; $\mathrm{V}=$ volume da solução $(\mathrm{mL}) ; \mathrm{e} \mathrm{M}=$ massa da amostra de terra (g).

Isotermas de adsorção ([Se $]_{\text {eq }}$ em função [Se $]_{\mathrm{ads}}$ ) foram construídas a partir dos resultados experimentais, e a adsorção de Se foi comparada com aquela estimada pela isoterma de Langmuir $A d s=\left(K_{L} C_{e q} A d s_{e q}\right) /$ $\left(1+K_{L} C_{e q}\right)$, em que $K_{L}$ é o parâmetro relacionado com a afinidade do solo pelo Se $\left(\mathrm{L} \mathrm{kg}^{-1}\right)$ e $\mathrm{Ads}_{\text {máx }}$ é a máxima capacidade de adsorção de $\operatorname{Se}\left(\mathrm{mg} \mathrm{kg}^{-1}\right)$.

A equação potencial utilizada para descrever o fenômeno de adsorção de Se foi a de Freundlich $A d s=K_{f} C_{e q}{ }^{n}$, em que $K_{f}$ é o coeficiente de Freundlich, intercepto que indica a capacidade do solo em reter um soluto, e n é um parâmetro adimensional, relacionado com a declividade da curva e que indica a afinidade do solo pelo soluto.

As isotermas de Langmuir e de Freundlich foram ajustadas aos resultados de adsorção de Se pelo programa Fitfunc (Barrow, 1987), que emprega a otimização não-linear dos quadrados mínimos e não exige a linearização da isoterma, o que evita tanto a introdução de mudanças na distribuição dos erros quanto a aquisição de parâmetros influenciados (Goldberg, 1995).

\section{RESULTADOS E DISCUSSÃO}

\section{Isotermas de adsorção}

Todos os solos apresentaram incrementos na adsorção, à medida que doses maiores do soluto foram adicionadas (Figura 1). Balistrieri \& Chao (1987) mostraram que o aumento da concentração de selenito na solução de equilíbrio resultou em incrementos decrescentes da adsorção do ânion em goethita. Em amostras de solos tropicais (LVwf, LAw e NVef), Casagrande et al. (2003) investigaram a adsorção dos ânions sulfato e fosfato, utilizando doses semelhantes às utilizadas para Se, e também encontraram incrementos decrescentes da adsorção desses elementos.

O formato das isotermas, principalmente sua inclinação inicial, é conveniente para descrever o comportamento dos sítios de superfície (Giles et al.,1974; Hinz, 2001). Para a maioria dos solos (LVA3, LV-1, LV-2, LVef, LVwf, LA-1, LA-2 e LAwf), o incremento na adsorção de Se foi decrescente e descrito por isotermas do tipo-L (Giles et al., 1974) (Figura 1), sendo muito freqüentes em estudos de adsorção, indicando que, com o aumento da dose adicionada, os sítios mais ativos para a adsorção de Se são ocupados (Sposito, 1989). Com a diminuição da quantidade de 
sítios, a reação de adsorção torna-se mais difícil de ocorrer, ocasionando a diminuição da inclinação da curva (Figura 1). Após a saturação dos sítios mais ativos, mecanismos não-preferenciais podem ter atuado no processo de adsorção. A provável existência de dois mecanismos de adsorção foi notada por Dhillon \& Dhillon (1999), ao avaliarem a adsorção de selenito em oito solos seleníferos da Índia. Peak (2006) notou que mecanismos de adsorção do tipo esfera interna (alta energia) e esfera externa (baixa energia) atuaram juntos na adsorção de selenito em oxihidróxidos de $\mathrm{Al}$. Independentemente da existência de mais de um mecanismo de adsorção, o LVef e o LAwf exibiram grande potencial para a adsorção de Se, confirmado pelas curvas com maiores inclinações iniciais (Figura 1).
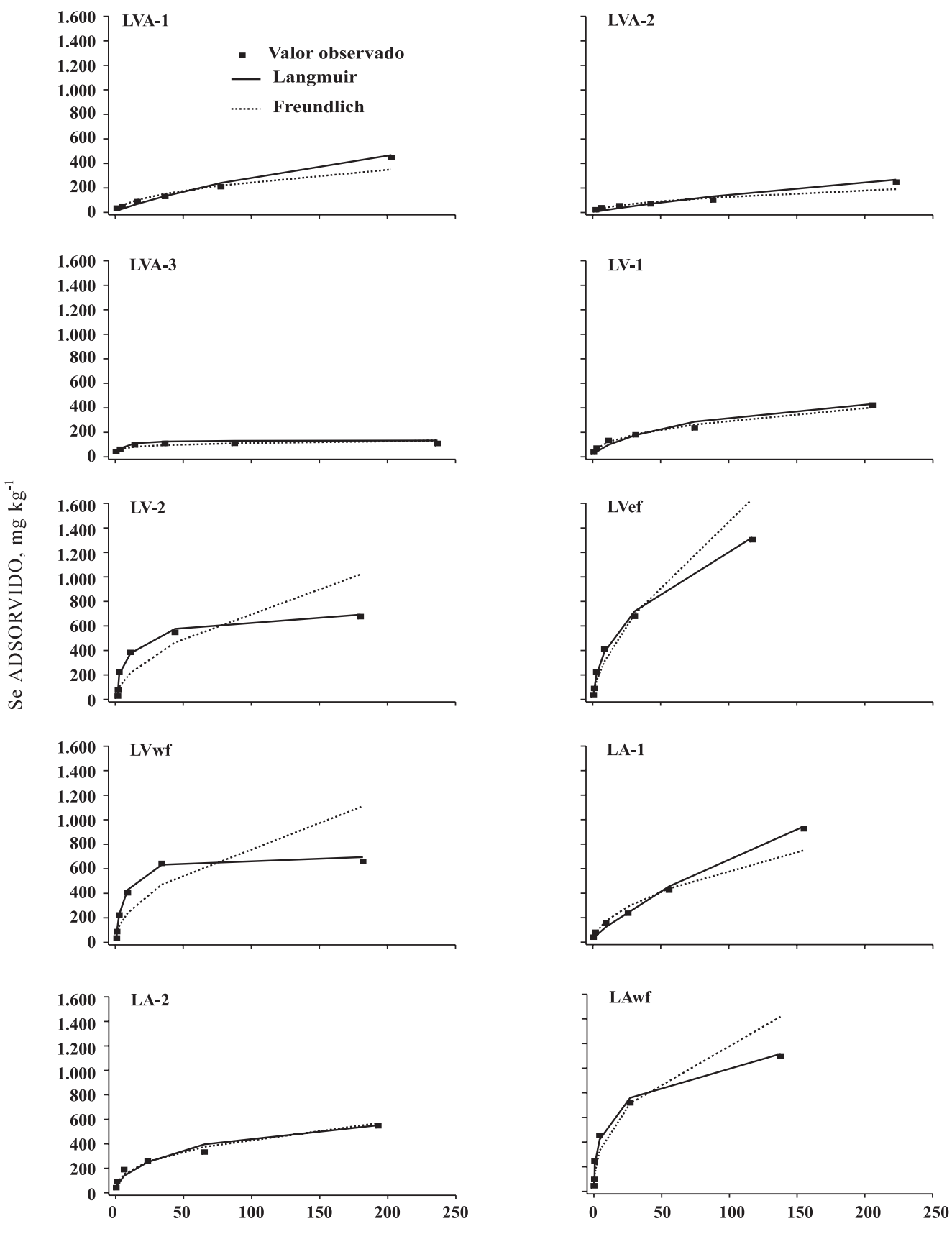

Se EM SOLUÇÃO DE EQUILÍBRIO, mg L

Figura 1. Isotermas de adsorção para os Latossolos Vermelho-Amarelos (LVA-1, LVA-2 e LVA-3), Latossolos Vermelhos (LV-1 e LV-2) e Latossolo Vermelho eutroférrico (LVef): pontos correspondem aos resultados experimentais; linhas contínuas e seccionadas correspondem aos ajustes pelos modelos de Langmuir e Freundlich, respectivamente. 
Isotermas do tipo-C foram obtidas para o LVA-1 e LVA-2 (Figura 1). Diferentemente das do tipo-L, estas isotermas mantiveram a inclinação inicial, sugerindo que o número de sítios disponíveis para a adsorção de Se permaneceu constante ou foi aumentado conforme a adição de maiores concentrações do soluto (Sposito, 1989). Nas isotermas do tipo-C, há afinidade constante do adsorvato pelo adsorvente, e os íons distribuem-se na interface sólido-solução sem nenhuma ligação específica (Meurer, 2000). Soares et al. (2005) observaram que isotermas do tipo- $\mathrm{C}$ descreveram a adsorção de boro em amostras de um Latossolo Vermelho, um Latossolo Amarelo e um Nitossolo Vermelho.

\section{Parâmetros obtidos a partir dos modelos de Langmuir e Freundlich}

Com base nos valores dos coeficientes de determinação obtidos para as equações de Langmuir $\left(R^{2}>0,99\right)$ e de Freundlich $\left(0,68<R^{2}<0,99\right)$ (Quadro 2), verificou-se que ambas as equações descreveram adequadamente a adsorção de Se nos solos. Em virtude da melhor aderência dos resultados experimentais ao modelo (Figura 1), a isoterma de Langmuir foi mais adequada e apontou para o provável predomínio de selenito na solução do solo. Em amostras de solos tropicais, Goh \& Lim (2004) verificaram que a adsorção de selenito foi mais bem descrita pelo modelo de Langmuir, enquanto a adsorção de selenato foi mais bem reproduzida pelo modelo de Freundlich.

A adsorção máxima de Se (parâmetro Ads máx de Langmuir) variou de 134,5 (LVA-3) a 2.245,8 mg kg-1 (LA-1) (Quadro 2). Os menores valores de Ads máx estão de acordo com a literatura (Singh et al., 1981; Dhillon \& Dhillon, 1999), mas os altos valores de Ads $_{\text {máx }}$ encontrados para o LVA-1, LVef, LA-1 e LAwf foram atípicos. Trabalhando com oito solos seleníferos da Índia e utilizando doses que variaram de $1 \mathrm{a}$ $100 \mathrm{mg} \mathrm{L}^{-1}$ de selenito, Dhillon \& Dhillon (1999) encontraram valores de Ads $s_{\text {máx }}$ que variaram de 109,17 a $460,83 \mathrm{mg} \mathrm{kg}^{-1}$, na faixa de reação alcalina, e de 161,29 a 454,55 mg kg-1, na faixa de reação ácida. Segundo estes autores, a elevada capacidade de adsorção de Se apresentada por solos alcalinos resultou da presença de elevados teores de óxidos de ferro mal cristalizados. Singh et al. (1981) estudaram a adsorção de Se em cinco solos com diferentes atributos e encontraram valores de $\mathrm{Ads}_{\text {máx }}$ que variaram de $68,0 \mathrm{mg} \mathrm{kg}^{-1}$, em solo orgânico, a 242,5 mg kg-1 em solo salino. O LVef e o LAwf também apresentaram valores altos de $\mathrm{Ads}_{\text {máx }}$ (Quadro 2). Esses resultados já eram esperados, uma vez que as isotermas destes solos apresentaram grande inclinação inicial (Figura 1).

Os valores encontrados para $\mathrm{K}_{\mathrm{L}}$ estiveram de acordo com os da literatura (Dhillon \& Dhillon, 1999, 2000). Elevados valores de $\mathrm{K}_{\mathrm{L}}$ apontaram o LVA-3 e o LVwf como os solos de maior afinidade por Se (Quadro 2). Conforme Parfitt (1978), ânions mostram maior afinidade por superfícies oxídicas. Oxihidróxidos amorfos de $\mathrm{Fe}$ [Fe $\mathrm{Fe}_{\mathrm{ox}}$ (Tabela 1)] têm maior afinidade por Se do que óxidos de manganês [MnO (Quadro 1)] (Balistrieri \& Chao, 1990).

$\mathrm{Na}$ ausência de evidências consistentes sobre a natureza dos mecanismos de adsorção, os coeficientes $\mathrm{K}_{\mathrm{f}}$ e $\mathrm{n}$ fornecidos pela equação potencial de Freundlich são considerados bons parâmetros descritivos (Buchter et al., 1989). A utilização do $\mathrm{K}_{\mathrm{f}}$ permite identificar a capacidade de um solo reter determinado soluto. $\mathrm{O}$ LVef e os Latossolos ácricos (LVwf e LAwf) apresentaram os maiores valores de $\mathrm{K}_{\mathrm{f}}$ e grande potencial adsortivo, ratificando o comportamento de suas curvas e os resultados anteriormente descritos para a $\mathrm{Ads}_{\text {máx }}$.

Quadro 2. Parâmetros quantitativos da adsorção de Se por Latossolos, obtidos do ajuste das isotermas de Langmuir e Freundlich

\begin{tabular}{|c|c|c|c|c|c|c|}
\hline \multirow{2}{*}{ Solo } & \multicolumn{2}{|c|}{ Parâmetros de Langmuir } & \multirow{2}{*}{$\mathbf{R}^{2}$} & \multicolumn{2}{|c|}{ Parâmetros de Freundlich } & \multirow{2}{*}{$\mathbf{R}^{2}$} \\
\hline & Adsmáx & $\mathbf{K}_{\mathbf{L}}$ & & $\mathbf{K}_{\mathbf{f}}$ & $\mathbf{n}$ & \\
\hline & $\mathrm{mg} \mathrm{kg}^{-1}$ & $\mathrm{~L} \mathrm{~kg}^{-1}$ & & $\mathrm{~L} \mathrm{~kg}^{-1}$ & & \\
\hline LVA-1 & 1070,9 & 0,004 & 0,99 & 26,2 & 0,49 & 0,96 \\
\hline LVA-2 & 787,3 & 0,002 & 0,99 & 13,7 & 0,49 & 0,95 \\
\hline LVA-3 & 134,5 & 0,326 & 0,99 & 52,1 & 0,17 & 0,87 \\
\hline LV-1 & 586,0 & 0,014 & 0,99 & 43,3 & 0,41 & 0,99 \\
\hline LV-2 & 736,3 & 0,085 & 0,99 & 56,5 & 0,58 & 0,70 \\
\hline LVef & 1786,8 & 0,024 & 0,99 & 77,9 & 0,64 & 0,93 \\
\hline LVwf & 713,4 & 0,214 & 0,99 & 77,5 & 0,51 & 0,77 \\
\hline LA-1 & 2245,8 & 0,005 & 0,99 & 52,2 & 0,53 & 0,98 \\
\hline LA-2 & 670,7 & 0,024 & 0,99 & 75,5 & 0,38 & 0,96 \\
\hline LAwf & 1239,2 & 0,065 & 0,99 & 180,1 & 0,42 & 0,89 \\
\hline
\end{tabular}

$\mathrm{R}^{2}$ : coeficiente de determinação. 
Embora o fenômeno de adsorção de Se pelos Latossolos tenha sido bem ajustado às isotermas do tipo-L e tipo- $\mathrm{C}$, as concentrações inicialmente empregadas parecem ter sido excessivas, pois as isotermas apresentaram curvatura logo nas primeiras doses para a maioria dos solos, o que pôde ser confirmado pelos valores de $\mathrm{n}$, que foram inferiores a 1 (Quadro 2). Os baixos valores de n encontrados neste estudo mostraram que o processo de adsorção de Se ocorreu principalmente em sítios de baixa energia. Utilizando doses de Se que variaram de 0,01 a $100 \mathrm{mg} \mathrm{L}^{-1}$, Buchter et al. (1989) encontraram valor de $n$ igual a 0,98 por meio da equação de Freundlich. Além disso, desvios da linearidade, identificados por valores de $\mathrm{n}$ diferentes de 1 , provavelmente descreveram situações em que os sítios mais ativos da superfície sólida tornaram-se ocupados, dificultando a adsorção adicional, ou à mesma taxa, dos elementos em solução (Buchter et al., 1989; Sposito, 1989), como mostraram as curvas tipo-L (Figura 1).

Não houve correlação significativa entre os atributos dos solos e os parâmetros de adsorção derivados da isoterma de Langmuir ( $\mathrm{Ads}_{\text {máx }}$ e $\mathrm{K}_{\mathrm{L}}$ ) (Quadro 3). A CTA foi o único atributo que apresentou correlação significativa $\operatorname{com~} \mathrm{K}_{\mathrm{f}}\left(\mathrm{r}=0,64^{*}\right)$ e $\operatorname{com} n$ $\left(r=0,57^{*}\right)$. Mesmo que a formação de complexos de esfera-interna seja o mecanismo predominante na adsorção de Se (Parfitt, 1978; Balistrieri \& Chao, 1990), as correlações entre os coeficientes de Freundlich $\left(\mathrm{K}_{\mathrm{f}}\right.$ e $\left.\mathrm{n}\right)$ e a CTA constituem possível indicativo de que a formação de complexos de esferaexterna, por meio de mecanismos de adsorção eletrostática, também é passível de ocorrência. O teor de argila também correlacionou-se com o coeficiente de afinidade de Freundlich $\mathrm{K}_{\mathrm{f}}\left(\mathrm{r}=0,42^{*}\right)$, o que está de acordo com Christensen et al. (1989), para quem a fração argila foi a principal responsável pela adsorção de Se em solos.

O coeficiente $n$ de Freundlich também correlacionouse com a capacidade de troca catiônica total $\left[\mathrm{CTC}_{\mathrm{t}}\right.$ $\left.\left(\mathrm{r}=0,72^{* *}\right)\right]$ e efetiva $\left[\mathrm{CTC}_{\mathrm{e}}\left(\mathrm{r}=0,58^{*}\right)\right]$ e com os teores de $\mathrm{CO}\left(\mathrm{r}=0,63^{* *}\right)$. Admitindo o predomínio de espécies aniônicas de Se em solução, não se observou relação causa-efeito entre a CTC e o coeficiente $n$. Provavelmente, a correlação com a CTC deve ser resultado da influência do $\mathrm{CO}$, que contribui para 50 a $95 \%$ da CTC dos horizontes superficiais de solos altamente intemperizados (Soares \& Alleoni, 2007). A correlação entre os valores de $\mathrm{n}$ e $\mathrm{CO}$, se, por um lado, contradiz a preferência da adsorção aniônica por superfícies minerais (Dhillon \& Dhillon, 1999), por outro, justifica o ajuste dos resultados experimentais ao modelo de Freundlich, que superestimou a capacidade adsortiva naqueles solos com altos valores de CO (Quadro 1), como pode ser observado por meio de suas isotermas (Figura 1), muito mais linearizadas e com inclinação maior do que aquelas ajustadas pelo modelo de Langmuir.

\section{CONCLUSÕES}

1. Os modelos de Langmuir e de Freundlich adequaram-se bem aos resultados experimentais da adsorção de Se pelos Latossolos, em toda a faixa de concentração estudada.

Quadro 3. Coeficientes de correlação de Pearson entre atributos dos Latossolos e os parâmetros de adsorção de Se

\begin{tabular}{|c|c|c|c|c|}
\hline \multirow{2}{*}{ Atributo do Latossolo } & \multicolumn{2}{|c|}{ Parâmetros de Langmuir } & \multicolumn{2}{|c|}{ Parâmetros de Freundlich } \\
\hline & Ads sáx & $\mathbf{K}_{\mathrm{L}}$ & $\mathbf{K}_{\mathbf{f}}$ & $\mathbf{n}$ \\
\hline & $\mathrm{mg} \mathrm{kg}^{-1}$ & $\mathrm{~L} \mathrm{~kg}^{\cdot 1}$ & $\mathrm{~L} \mathrm{~kg}^{-1}$ & \\
\hline Argila & $0,16 \mathrm{~ns}$ & $0,18 \mathrm{~ns}$ & $0,42^{*}$ & $0,57 \mathrm{~ns}$ \\
\hline $\mathrm{pH}_{\mathrm{H} 2 \mathrm{O}}$ & $0,35 \mathrm{~ns}$ & $-0,29 \mathrm{~ns}$ & $0,04 \mathrm{~ns}$ & $0,34 \mathrm{~ns}$ \\
\hline $\mathrm{pH}_{\mathrm{KCl}}$ & $0,43 \mathrm{~ns}$ & $-0,26 \mathrm{~ns}$ & $0,08^{\mathrm{ns}}$ & $0,38 \mathrm{~ns}$ \\
\hline $\mathrm{CTC}_{\mathrm{t}}$ & $0,40 \mathrm{~ns}$ & $-0,01 \mathrm{~ns}$ & $0,36^{\mathrm{ns}}$ & $0,72^{* *}$ \\
\hline $\mathrm{CTC}_{\mathrm{e}}$ & $0,47 \mathrm{~ns}$ & $-0,21 \mathrm{~ns}$ & $0,03 \mathrm{~ns}$ & $0,58^{*}$ \\
\hline CTA & $0,24 \mathrm{~ns}$ & $0,16 \mathrm{~ns}$ & $0,64^{*}$ & $0,57^{*}$ \\
\hline $\mathrm{CO}$ & $0,49 \mathrm{~ns}$ & $0,01 \mathrm{~ns}$ & $0,25 \mathrm{~ns}$ & $0,63^{* *}$ \\
\hline $\mathrm{Fe}_{\mathrm{DCB}}$ & $0,15^{\mathrm{ns}}$ & $0,21 \mathrm{~ns}$ & $0,43 \mathrm{~ns}$ & $0,52 \mathrm{~ns}$ \\
\hline $\mathrm{Fe}_{o x}$ & $0,12 \mathrm{~ns}$ & $0,25 \mathrm{~ns}$ & $0,35^{\mathrm{ns}}$ & $0,46 \mathrm{~ns}$ \\
\hline $\mathrm{Al}_{\mathrm{ox}}$ & $0,14 \mathrm{~ns}$ & $0,23 \mathrm{~ns}$ & $0,46 \mathrm{~ns}$ & $0,49 \mathrm{~ns}$ \\
\hline $\mathrm{Mn}_{\mathrm{ox}}$ & $0,38 \mathrm{~ns}$ & $0,02 \mathrm{~ns}$ & $0,15^{\mathrm{ns}}$ & $0,51 \mathrm{~ns}$ \\
\hline $\mathrm{MnO}$ & $0,41 \mathrm{~ns}$ & $-0,01 \mathrm{~ns}$ & $0,14 \mathrm{~ns}$ & $0,51 \mathrm{~ns}$ \\
\hline
\end{tabular}

Significância estatística: ${ }^{\mathrm{ns}}$ não-significativo; ${ }^{*} \mathrm{p}<0,10 ;{ }^{* *} \mathrm{p}<0,05$. 
2. O modelo de Langmuir apresentou melhor ajuste dos resultados experimentais do que o modelo de Freundlich.

3. Os Latossolos Vermelho-Amarelos LVA-1 e o LVA-2 apresentaram isotermas do tipo-C, enquanto os demais Latossolos apresentaram isotermas tipo-L.

4. Os parâmetros quantitativos de adsorção ( $\mathrm{Ads}_{\text {máx }}$ de Langmuir e $\mathrm{K}_{\mathrm{f}}$ de Freundlich) indicaram grande potencial de imobilização de Se no Latossolo Vermelho eutroférrico, no Latossolo Amarelo acriférrico e no Latossolo Vermelho acriférrico.

5. De acordo com o parâmetro qualitativo de adsorção de Langmuir $\left(\mathrm{K}_{\mathrm{L}}\right)$, obteve-se a seguinte ordem decrescente de afinidade pelo Se: LVA-3, LVwf, LV-2, LAwf, LVef = LV-2, LV-1, LA-1, LVA-1 e LVA-2.

\section{LITERATURA CITADA}

ALLEONI, L.R.F.; CAMARGO, O.A. \& CASAGRANDE, J.C. Isotermas de Langmuir e de Freundlich na descrição da adsorção de boro em solos altamente intemperizados. Sci. Agric., 55:379-387, 1998.

ALVES, M.E. \& LAVORENTI, A. Sulfate adsorption and its relationships with properties of representative soils of the São Paulo State, Brazil. Geoderma, 118:89-99, 2004.

BALISTRIERI, L.S. \& CHAO, T.T. Adsorption of selenium by amorphous iron oxyhydroxide and manganese dioxide. Geochim. Cosmochim. Acta, 54:739-751, 1990.

BALISTRIERI, L.S. \& CHAO, T.T. Selenium adsorption by goethite. Soil Sci. Soc. Am. J., 51:1145-1151, 1987.

BAR-YOSEF, B. \& MEEK, D. Selenium sorption by kaolinite and montmorillonite. Soil Sci., 144:11-19, 1987.

BARROW, N.J. Reactions with variable-charge soils. Dordrecht, Martinus Nijhoff Publishers, 1987. 191p.

BUCHTER, B.; DAVIDOFF, B.; AMACHER, M.C.; HINZ, C.; ISKANDAR, I.K. \& SELIM, H.M. Correlation of Freundlich $\mathrm{K}_{\mathrm{d}}$ and $\mathrm{n}$ retention parameters with soils and elements. Soil Sci., 148:370-379, 1989.

CAMARGO, O.A.; MONIZ, A.C.; JORGE, J.A. \& VALADARES, J.M.A.S. Métodos de análise química, mineralógica e física de solos do Instituto Agronômico de Campinas. Campinas, Instituto Agronômico de Campinas, 1986. 94p. (Boletim Técnico, 106).

CASAGRANDE, J.C.; ALLEONI, L.R.F.; CAMARGO, O.A. \& BORGES, M. Adsorção de fosfato e sulfato em solos com cargas variáveis. R. Bras. Ci. Solo, 27:51-59, 2003.

CHRISTENSEN, B.T.; BERTELSEN, F. \& GISSEL-NIELSEN, G. Selenite fixation by soil particle-size separates. Eur. J. Soil Sci., 40:641-647, 1989.

DHILLON, K.S. \& DHILLON, S.K. Adsorption-desorption reactions of selenium in some soils of India. Geoderma, 93:19-31, 1999
DHILLON, K.S. \& DHILLON, S.K. Distribution and management of seleniferous soils. Adv. Agron., 79:119184, 2003.

DHILLON, K.S. \& DHILLON, S.K. Selenium adsorption in soils as influenced by different anions. Plant Nutr. Soil Sci., 163:577-582, 2000.

FISHBEIN, L. Selenium. In: MERIAN, E., ed. Metals and their compounds in the environment: Occurrence, analysis and biological relevance. Weinheim, VHC, 2001. p.1309-1342.

FORD, R.G.; SCHEINOST, A.C. \& SPARKS, D.L. Frontiers in metal sorption/precipitation mechanisms on soil mineral surfaces. Adv. Agron., 74:41-62, 2001

GAMBRELL, R.P. Manganese. In: BIGHAM, J.M., ed. Methods of soil analysis. Part 3. Chemical methods. Madison, Soil Science Society of America; America Society of Agronomy, 1996. p.665-682.

GEERING, H.R.; CARY, E.E.; JONES, L.H.P. \& ALLAWAY, W.H. Solubility and redox criteria for the possible forms of selenium in soils. Soil Sci. Soc. Am. J., 32:36-40, 1968.

GILES, C.H.; SMITH, D. \& HUITSON, A. A general treatment and classification of the solute adsorption isotherm. I. Theoretical. J. Colloid Interf. Sci., 47:755-765, 1974.

GILLMAN, G.P. A proposed method for the measurement of exchange properties of highly weathered soils. Aust. J. Soil Res., 17:129-139, 1979.

GIRLING, C.A. Selenium in agriculture and the environment. Agric., Ecosyst. Environ., 11:37-65, 1984.

GOH, K.H. \& LIM, T.T. Geochemistry of inorganic arsenic and selenium in a tropical soil: Effect of reaction time, $\mathrm{pH}$, and competitive anions on arsenic and selenium adsorption. Chemosphere, 55:849-859, 2006.

GOLDBERG, S. Adsorption models incorporated into chemical equilibrium models. In: LOEPPERT, R.H.; SCHWAB, A.P. \& GOLDBERG, S., eds. Chemical equilibrium and reaction models. Madison, Soil Science Society of America, 1995. p.75-95 (Special Publication, 42)

HINZ, C. Description of sorption data with isotherm equations. Geoderma, 99:225-243, 2001

LISK, D.J. Trace metals in soils, plants and animals. Adv. Agron., 24:267-311, 1972.

MEURER, J.E. Fundamentos de química do solo. Porto Alegre, Gênesis, 2000. 174p.

NAKAMARU, Y.; TAGAMI, K. \& UCHIDA, S. Distribution coefficient of selenium in Japanese agricultural soils. Chemosphere, 58:1347-1354, 2005.

NEAL, R.H. Selenium. In: ALLOWAY, B.J. Heavy metals in soils. 2.ed. New York, Wiley, 1995. p.260-283.

PARFITT, R.L. Anion adsorption by soils and soil materials. Adv. Agron., 30:1-50, 1978. 
PEAK, D. Adsorption mechanisms of selenium oxyanions at the aluminum oxide/water interface. J. Colloid Interf. Sci., 303:337-345, 2006.

SAEKI, K.; MATSUMOTO, S. \& TATSUKAWA, R. Selenite adsorption by manganese oxides. Soil Sci., 160:265-272, 1995.

SINGH, M.; SINGH, N. \& RELAN, P.S. Adsorption and desorption of selenite and selenate selenium on different soils. Soil Sci., 132:134-141, 1981.

SOARES, M.R. Coeficiente de distribuição $\left(\mathrm{K}_{\mathrm{d}}\right)$ de metais pesados em solos do Estado de São Paulo. Piracicaba, Escola Superior de Agricultura Luiz de Queiroz, 2004. 202p. (Tese de Doutorado)
SOARES, M.R.; ALLEONI, L.R.F. \& CASAGRANDE, J.C. Parâmetros termodinâmicos da reação de adsorção de boro em solos tropicais altamente intemperizados. Química Nova, 28:1014-1022, 2005.

SOARES, M.R. \& ALLEONI, L.R.F. Contribution of soil organic carbon to the ion exchange capacity of tropical soils. J. Sustainable Agric., 2007. (No Prelo)

SPARKS, D.L. Kinetics and mechanisms of chemical reactions at the soil mineral/water interface. In: SPARKS, D.L. Soil physical chemistry. 2.ed. New York, CRC Press, 1999. p.135-192.

SPOSITO, G. The chemistry of soils. New York, Oxford University Press, 1989. 276p. 